\title{
ReviewArticle
}

\section{Laboratory Diagnosis of Tuberculosis- an Update}

\author{
F Ahmed ${ }^{1}$, AM Hossain ${ }^{2}$, MAB Siddique ${ }^{3}$, NN Biswas ${ }^{4}$
}

\begin{abstract}
:
Annually about two million deaths occur globally due to tuberculosis (TB). Bangladesh ranks the sixth position among 22 highest burden TB countries in the world and also one of the 27 high multidrug resistant tuberculosis (MDR-TB) burden countries where about 70,000 people die every year due to TB. Among six key components of Stop TB Strategy (STS) Plan, the first one includes increase case notification of all forms of TB and improve diagnosis of new smear negative, extrapulmonary cases and TB in children by 2016 . As TB can affect any organ in human body, the TB cases are managed by any discipline in medical community. Unfortunately diagnostic accuracy is not satisfactory and is not only due to uniform unavailability of the latest diagnostic facilities but also due to inadequate knowledge of the professionals about currently available modern laboratory techniques to diagnose TB. Light-emitting diode (LED) microscopy with fluorescence (auramine-rhodamine staining) should be preferred than conventional microscopy with Zeihl-neelsen (acid fast) staining to identify TB bacilli. Mantoux test (MT) indicates only infection by TB bacilli, does not necessarily the active disease. It may be positive in latent TB and in BCG (Bacillus Calmette-Guerin) vaccinated cases. Antibodies from Lymphocyte Secretion or Antibodies in Lymphocyte Supernatant (ALS) assay can detect active TB cases within three days of sample collection. The test is very useful to diagnose TB in children where sputum collection is difficult. Interferon gamma release assay (IGRA) tests are not advocated in low and middle-income countries, typically those with a high TB and/or HIV burden. Anti TB IgG/IgM/IgA tests should be avoided because these are being misinterpreted by someone as active TB cases. Adenosine Deaminase Assay (ADA) is a reliable test to diagnose tuberculous pleural effusion together with other evidences. ADA in pleural fluid $<40 \mathrm{IU} / \mathrm{L}$ is considered negative for TB. The more the ADA level, the more possibility to be tuberculous effusion. Level $>100$ IU/L is highly specific for TB origin. Gene Xpert MTB/RIF, an Xpert test for mycobacterium tuberculosis (MTB) and Rifampicin (RIF) resistance, is used for rapid identification for TB bacilli, specially when MDR-TB is suspected, in human immunodeficiency virus ( HIV) infected cases and highly suspected sputum negative cases ( as a follow on test ) where microscopy frequently failed due to low bacterial load. The test exhibits high sensitivity and specificity for detecting pulmonary TB.
\end{abstract}

Key words: Tuberculosis Diagnosis.

\section{Introduction:}

TB continues to remain a major public health problem with nearly 9 million new active disease cases and nearly 2 million deaths occurring worldwide every year ${ }^{1}$. Geographicaly the burden of TB is highest in Asia ( $40 \%$ of total TB cases are in south east Asia) and Africa (26\% of all cases). Bangladesh ranks the sixth position among 22 highest burden TB countries in the

1. Dr. Faruk Ahammad. MBBS, FCPS, Associate Professor of Medicine. Faridpur Medical College, Faridpur.

2. Dr.Ahmed Manadir Hossain, MBBS, FCPS, Assistant Professor, Medicine, Faridpur Medical College, Faridpur.

3. Dr. Mohammad Abu Bakar Siddique, MBBS, MD, Assistant Professor, Dept. of Medicine, Faridpur Medical College, Faridpur.

4.Dr. Nipendra Nath Biswas, DLO, FCPS (ENT) Assistant Professor, Dept. of ENT, Faridpur Medical College. Faridpur.

Address of correspondence :

Dr. Faruk Ahammad. MBBS, FCPS, Associate Professor of

Medicine, Faridpur Medical College, Faridpur.

Cell: +88-01817011894, E-mail: farukahammad26@yahoo.com world. Here about 70,000 people die every year due to $\mathrm{TB}^{2}$. The country is also one of the 27 high MDR-TB burden countries. According to WHO estimate, MDRTB among all new cases are $2.2 \%$ and among previously treated cases are $15 \%$. Among patients failing re-treatment regimen show up to $88 \%$ MDR-TB. In 1965 TB services were started in Bangladesh in 44 TB clinics, 8 segregation hospitals and 4 TB hospitals; now expanded to 460 upazillas since $1998^{3}$. The National Tuberculosis Programme (NTP) in Bangladesh adopted 'WHO Global and Regional Stop TB Strategy' and will continue in the current 2012-16 plan. The programme has adopted the 'Universal Access' for quality diagnosis and treatment of all TB cases in the community to achieve the millennium development goal (MDG) for TB such as halving the death and prevalence of TB by 2015 . The programme has also target to eliminate TB from Bangladesh by the year 2050. Among six key components of STS plan, the 
first one includes increase case notification of all forms of TB and improve diagnosis of new smear negative, extrapulmonary cases and TB in children by 2016. To meet this key component, the diagnostic procedures are the main tools. In this article we will discuss the available diagnostic procedures for $\mathrm{TB}$, pros and cons of those.

\section{Methodology: \\ Pubmed was searched in April 2015 for all English language publications including the search terms 'Laboratory diagnosis of tuberculosis', 'LED- Fluorescence Microscopy', 'Interferon-gamma release assays (IGRAs)', 'Nucleic acid amplification test (NAAT)', 'Adenosine Deaminase Assay' and 'Gene- expert', from 1990 onwards. The full articles for selected identified records that were thought to be potentially relevant were collected. The references in these articles were also checked for additional relevant information.}

\section{Laboratory investigations of tuberculosis:}

Primarily a pathogen of the respiratory system, aerobic Mycobacterium tuberculosis complex (MTBC) infects the lungs via the inhalation of infected aerosol droplets generated by people with pulmonary disease through coughing. MTBC consists of $\mathrm{M}$. tuberculosis, $\mathrm{M}$. bovis, M. africanum, M. caprae, M. microti, M. canettii and M. pinnipedii, all of which share genetic homology, with no significant variation between sequences, although differences in phenotypes are present ${ }^{4}$. Mycobacterium tuberculosis was first discovered in 1882 by Robert Koch and is one of almost 200 mycobacterial species which have been detected by molecular techniques ${ }^{5}$. Since it's discovery days after days are being expended to save the humanity from it's devastating attack by inventing newer laboratory methods and drugs. Followings are the widely used laboratory tests to diagnose the TB cases.

\section{Microbiological tests:}

(a) Culture: a definitive diagnosis of tuberculosis can only be made by culturing Mycobacterium tuberculosis organisms from a specimen taken from the patient (ie, sputum, pus, CSF, biopsied tissue etc.). A diagnosis made other than by culture may only be classified as "probable" or "presumed". Traditional culture in the Löwenstein-Jensen media needs more time (4-8 weeks). New automated systems that are faster and more accurate include the MB/BacT, BACTEC 9000 MB, BACTEC 460 TB, VersaTREK, Mycobacterial Growth Indicator Tube (MGIT) and the Microscopic Observation Drug Susceptibility (MODS) assay culture methods ${ }^{6}$. BACTEC needs about one week and MODS needs about 10 days for mycobacterial tuberculosis growth. (b) Microscopic examination: it is the widely accepted procedure to find out the pathogen due to long time needed for culture. The preferred method is the LEDFM with fluorescence (auramine-rhodamine) staining, which is more sensitive than conventional ZiehlNeelsen staining ${ }^{7}$. Fluorochrome stains, such as single auramine $\mathrm{O}$ or the combination of auramine-rhodamine $\mathrm{B}$, is quicker to perform and more sensitive. The auramine $\mathrm{O}$ fluorescence is enhanced on binding to DNA or RNA as opposed to the carbol fuchsin dye that stains the cell membrane in $\mathrm{ZN}$ staining.

(c) Polymerase chain reaction (PCR) or gene probe tests can distinguish $M$. tuberculosis from other mycobacteria. Even if sputum smear is negative, tuberculosis must be considered in PCR positive cases and is only excluded after negative cultures.

\section{Immunological tests:}

(a) ALS assay- this is an immunological assay to detect active diseases like tuberculosis, cholera, typhoid etc. International Centre for Diarrhoeal Disease Research, Bangladesh (ICDDR,B) innovated the test first and recently ALS assay nods the scientific community as it is used for rapid diagnosis of tuberculosis. The principle is based on the secretion of antibody from in vivo activated plasma $B$ cells found in blood circulation for a short period of time in response to TB-antigens during active $\mathrm{TB}$ infection rather than latent $\mathrm{TB}$ infection. It can be used both in pulmonary and extrapulmonary TB diagnosis. The test is very helpful in TB in children where sputum collection is difficult and not satisfactory. This is a novel diagnostic method in the diagnosis and subsequent monitoring of treatment effectiveness of paediatric $\mathrm{TB}^{8}$. It takes only three days to give the result. The test can't be done within 40 days of MT. It should be noted that this is a complimentary test to other tests for TB.

(b) Mantoux test (MT)/Tuberculin Skin Test (TST)positive test indicates infection by TB bacilli, doesn't exclude active disease cases from latent cases and inconclusive in BCG vaccinated people. In developed countries where TB infection is less and BCG vaccination is not routinely done, the positive MT has a rule for further evaluation. United States' (US) Centre for disease control (CDC) classify tuberculin reactors as follows-

An induration (palpable raised hardened area of skin) of more than 5-15 mm (depending upon the person's risk factors) to 10 Mantoux units is considered a positive result, indicating TB infection. 
(1) $5 \mathrm{~mm}$ or more is positive in-

* HIV-positive person

* Recent contacts of TB case

* Persons with nodular or fibrotic changes on chest x-ray (CXR) consistent with old healed TB

*Patients with organ transplants and other immunosuppressed patients

(2) $10 \mathrm{~mm}$ or more is positive in-

*Recent arrivals (less than 5 years) from highprevalent countries

* Injection drug users

* Residents and employees of high-risk congregate settings (e.g., prisons, nursing homes, hospitals, homeless shelters, etc.)

* Mycobacteriology lab personnel

* Persons with clinical conditions that place them at high risk (e.g., diabetes, prolonged corticosteroid therapy, leukemia, end-stage renal disease, chronic malabsorption syndromes, low body weight, etc.)

* Children less than 4 years of age, or children and adolescents exposed to adults in high-risk categories

(3) $15 \mathrm{~mm}$ or more is positive in-

* Persons with no known risk factors for TB

Tuberculin skin test (TST) conversion: a tuberculin test conversion is defined as an increase of $10 \mathrm{~mm}$ or more within a 2-year period of negative test (4 $\mathrm{mm}$ or less), regardless of age. When a reaction is $5-9 \mathrm{~mm}$, an increase of $6 \mathrm{~mm}$ in this period is considered as conversion. TST conversion is considered presumptive evidence of acquired latent TB infection and potential risk for progression to active tuberculosis disease.

BCG vaccination and MT: There is disagreement on the use of the MT for people who have been immunized with BCG vaccination. The US recommendation is that in administering and interpreting the MT, previous BCG vaccination should be ignored; the United Kingdom (UK) recommendation is that interferon- $\gamma$ tests should be used to interpret positive tuberculin tests. The UK does not recommend serial tuberculin skin testing (a key part of the US strategy) in people who have had BCG vaccination'. In general the US approach is likely to result in more false positives and more unnecessary treatment with potentially toxic drugs; the UK approach is as sensitive in theory and should also be more specific, because of the use of interferon- $\gamma$ tests.

Under the US recommendations, diagnosis and treatment of latent tuberculosis infection (LTBI) is considered for any BCG-vaccinated person whose skin test is $10 \mathrm{~mm}$ or greater, if any of these circumstances are present:
* Was in contact with another person with infectious TB

* Was born or has resided in a high TB prevalence country

* Is continually exposed to populations where TB prevalence is high.

(c) IGRAs: These new developments in TB infection testing also measure a person's immune reactivity to $\mathrm{M}$. tuberculosis. IGRAs are based on the ability of the Mycobacterium tuberculosis antigens for early secretory antigen target 6 (ESAT-6) and culture filtrate protein 10 (CFP-10) to stimulate host production of interferon-gamma. As these antigens are not present in non-tuberculous mycobacteria (NTM) or in any BCG vaccine variant, these tests can distinguish mycobacterium tuberculosis from other mycobacteriosis and can diagnose LTBI specially those with BCG vaccinated. IGRAs require a single patient visit, results are available in $24-48 \mathrm{hrs}$.

Two IGRAs that have been approved by the U.S. Food and Drug Administration (FDA) are commercially available in the U.S:

* QuantiFERON®-TB Gold In-Tube test (QFT-GIT); * T-SPOT®.TB test (T-Spot)

Limitations of these tests: IGR As are expensive, special laboratory infrastructure and supplies, and adequately trained staffs are required. Children younger than 5 years of age, persons recently exposed to $M$. Tuberculosis and immunocompromised persons are not advised to use these tests.

\section{Adenosin Deaminase Assay (ADA) test:}

ADA is an enzyme which contributes in purin metabolism and converts adenosine to inosine. ADA is essential for proliferation and differentiation of lymphoid cells, especially $\mathrm{T}$ cells, and helps in the maturation of monocytes to macrophages. It seems ADA is an index for cellular immunity. Activity of this enzyme increases in TB, empyema, lymphoma and other chronic inflammatory conditions like Rheumatoid Arthritis (RA). Although there is no evidence to support the use of ADA tests for the diagnosis of pulmonary $\mathrm{TB}$, there is considerable evidence to support their use in pleural fluid samples for diagnosis of pleural TB, where sensitivity is very high and higher the pleural fluid ADA level, the more likely the patient is to have TB. Raised ADA in cerebrospinal fluid is also an indicator for TB meningitis ${ }^{10}$. The ADA assay is inexpensive, rapid and simple to perform and is of great value for the immediate diagnosis of tuberculous pleuritis while waiting for culture result and this has a positive impact on patient outcome. In countries with low incidence of TB the diagnostic cut off value of ADA level have to be different from countries with high incidence. Adenosine deaminase level of less than 
$40 \mathrm{U} / \mathrm{L}$ in adult patients excludes the tubercular etiology of exudative pleural effusion. In many cases of non-tuberculous exudative pleural effusions the ADA level can exceed the cut off values of tubercular disease (40 UI/L). Here other tests must be co-related. The best cut off value of pleural fluid ADA level in diagnosis of $\mathrm{TB}$ is $100 \mathrm{UI} / \mathrm{L}^{11}$.

\section{Nucleic Acid Amplification Test (NAAT):}

This is a heterogeneous group of tests that use either the PCR technique or Transcription mediated amplification (TMA) or other forms of nucleic acid amplification methods to detect mycobacterial nucleic acid. The two most common commercially available tests are the amplified mycobacterium tuberculosis direct test (AMTD, Gen-Probe) and Amplicor. NAAT test accuracy is superior when applied to respiratory samples as opposed to other specimens and the AMTD test appears to perform better than other currently available commercial tests ${ }^{10}$. The accuracy of the AMTD test as follows:

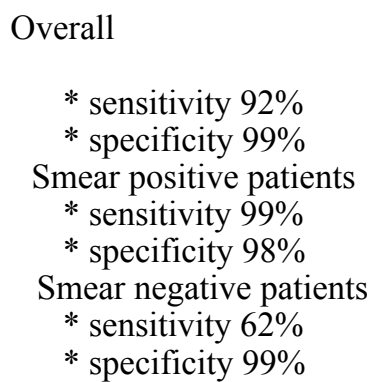

\section{Gene Xpert MTB/RIF test:}

The Xpert MTB/RIF is a cartridge-based, automated new molecular diagnostic test that can identify Mycobacterium tuberculosis DNA and resistance to Rifampicin (RIF) by NAAT. It uses a sputum sample and can give a result in less than 2 hours. It can also detect the genetic mutations associated with resistance to the drug Rifampicin ${ }^{12}$.

WHO recommended that the test should be used as the initial diagnostic test in individuals suspected of having MDR- TB or HIV associated TB. They also suggested that it could be used as a follow on test to microscopy in settings where MDR-TB and/or HIV is of lesser concern, especially in smear negative specimens, because of the lack of accuracy of smear microscopy ${ }^{13}$.

\footnotetext{
Disadvantages:

* The shelf life of the cartridges is only 18 months

* A very stable electricity supply is required

* The instrument needs to be recalibrated annually

* The cost of the test

* The temperature ceiling is critical ${ }^{14}$.
}

Advantages:

* Reliable when compared to sputum microscopy

* Short time test when compared with culture for RIF resistance.

\section{Discussion:}

Tuberculosis globally results in almost 2 million human deaths annually, with 1 in 4 deaths from tuberculosis being HIV/acquired immunodeficiency syndrome (AIDS)-related. Traditionally TB has been diagnosed by looking for evidence of TB bacteria either through the use of the chest X-ray, through sputum smear microscopy, or through the culturing of bacteria. Each of these TB tests has their disadvantages, one of the most significant for culture being the time that it takes and for sputum the issue of accuracy. Moreover diagnosis of extrapulmonary TB sometimes become difficult. In these regard newer techniques like LEDmicroscopy, Xpert test, ALS and ADA test are playing good roles and these tests are available in Bangladesh. Despite being more sensitive and taking less time to perform, fluorescent microscopy uses mercury vapor as the light source and so may pose a health risk to the lab operative. Furthermore, there is a requirement for a dark room; equipment and reagents are expensive. As a result small laboratories may be cost-compromised. Microscopy using either $\mathrm{ZN}$ or fluorochrome staining dyes may potentially give false-positive results due to cross contamination from many sources, such as the presence of Gram-positive-like Nocardia sp, due to the handling process, the transfer of AFB from slide to slide through immersion oil and misinterpretation of results by untrained lab personnel. In addition, light and fluorescent microscopy is not adequately sensitive for the detection of MDR-TB and cannot differentiate between MTBC and NTM. The introduction of LEDFM needs to be accompanied by appropriate training, quality management and monitoring of performance in the field. In clinical application, serological tests have not been used widely due to the insensitivity and low antigen specificity. The classic serologic test for tuberculosis is TST. However, this method is incapable of distinguishing active tuberculosis disease from pastsensitization of BCG, it has unknown predictive values and it allows cross-reaction with NTM. Two systems of whole-blood IGRA determination kits, T-SPOT. TB and QFNG-IT are to use with the positive TST cases. Although detection sensitivity is higher with IGRA testing kits in comparison with TST, these are not advocated in high TB prevalent countries like Bangladesh. Like TST and IGRAs, anti TB IgG/ $\operatorname{IgM} / \operatorname{IgA}$ tests are also nonspecific due to false positivity in old $\mathrm{TB}, \mathrm{BCG}$ vaccinated cases and in NTM. There is no guideline to use these immunoglobulin (Ig) tests to diagnose TB. One study shows, if $36 \mathrm{IU} / \mathrm{L}$ is taken as cut of limit, the sensitivity and specificity of ADA for tuberculosis in pleura is $100 \%$ and $77.7 \%$ respectively. More than $100 \mathrm{IU} / \mathrm{L}$ was exclusively seen in tubercular pleural effusion ${ }^{15}$. Another study found 40 IU $/ \mathrm{L}$ as a cut of 
limit for tuberculous pleural effusion where less than $40 \mathrm{IU} / 1$ in exudative pleural effusion is very unlikely to be tuberculous in origin. This study found $95 \mathrm{IU} / \mathrm{L}$ ADA as a strong evidence of tuberculous pleural effusion. ${ }^{11}$ It should be remembered that raised ADA in pleural fluid should not be the only parameter in TB diagnosis rather an adjunct with other findings such as pleural fluid cytology, where lymphocyte count is high. Lymphocyte : neutrophil ratio $>0.75$ in pleural fluid with raised ADA in pleural fluid increases the specificity of TB diagnosis. Esinophil $>10 \%$ in pleural fluid usually excludes the diagnosis of TB pleuritis. In TB pleural effusion, mesothelial cells are $<3 \%$ and in effusion due to RA lymphocytosis do not occur. ALS assay available in ICDDR,B detects active TB cases within three days of sample collection. The test become positive at early stage of the disease when clinical symptoms yet not prominent. So early case detection by ALS assay can prevent transmission of TB. Moreover, the test can be useful both in pulmonary and extrapulmonary cases. In children where sputum collection is difficult ALS assay is very helpful. The Xpert test is available at 26 sites including National Institute of Chest diseases and Hospital (NIDCH) in Bangladesh $^{2}$. The Gene Xpert MTB/RIF has been developed by the Foundation for Innovative New Diagnostics (FIND), who have partnered with the Cepheid Corporation and the University of Medicine and Dentistry of New Jersey. The Gene Xpert system was launched in 2004, and the development of the Gene Xpert MTB/RIF test, based on the Gene Xpert platform, was completed in 2008. The first clinical validation studies were carried out in 2009. A large scale demonstration project was then carried out in a number of different countries including South Africa and India to assess the implementation of the Gene Xpert MTB/RIF ${ }^{12,16}$. In December 2010, WHO endorsed the Gene Xpert MTB/RIF technology and released a recommendation and guidance for countries to incorporate the new test into their programs. For diagnosis of TB, although sputum microscopy is both quick and cheap, it is often unreliable particularly in HIV positive cases. Although culture gives a definitive diagnosis, to get the result usually takes weeks rather than the hours of the Xpert test. WHO did also emphasize that the test does not eliminate the need for conventional microscopy, culture and drug sensitivity testing, as these are still required to monitor treatment progress and to detect other types of drug resistance. The Gene Xpert MTB/RIF cannot be used for treatment monitoring, as it detects both live and dead bacteria.

\section{Conclusion:}

Although for diagnosis of TB, microscopic examination is still the first option and widely available in our country; this should be done with LED- microscope facility. Chest X-ray should be done in every sputum negative suspected pulmonary cases. Neither IGRAs nor the TST should be used for the diagnosis of active
TB disease. Anti TB IgG/IgM/IgA should be discarded as a TB tests. ALS assay is a good reliable complimentary test to diagnose early active TB, both pulmonary and extrapulmonary; specially in children in a short period. For TB pleuritis diagnosis, ADA test is a good option. When TB diagnosis and treatment become difficult; specially in HIV and suspected MDR cases, Xpert test must be done.

\section{References :}

1. Ahmad S. New approaches in the diagnosis and treatment of latent tuberculosis infection. Respir Res. 2010; 11(1): 169.

2. WHO global tuberculosis report 2014.

3. Revised Strategic Plan for National TB Control Plan(NTP) 20122016 in Bangladesh.

4. Talip BA, Sleator RD, Lowery CJ, Dooley JSG, Snelling WJ. An Update on Global Tuberculosis (TB). Infect Dis (Auckl). 2013; 6: $39-50$.

5. Palicova F. Mycobacterium: General Characteristics, Laboratory Detection, and Staining Procedures. In: Versalovic J, Carroll KC, Funke G, Jorgensen JH, Landry M, Warnock DW, editors. Manual of Clinical Microbiology. 10th ed. Vol. 1. Washington: ASM Press; 2011. p. 472-502.

6. Moore D, Evans C, Gilman R, Caviedes L, Coronel J, Vivar A, et al. Microscopic-observation drug-susceptibility assay for the diagnosis of TB. N Engl J Med.2006; 355 (15): 1539-50.

7. Steingart K, Henry M, Ng V, Hopewell PC, Ramsay A, Cunningham J,et al. Fluorescence versus conventional sputum smear microscopy for tuberculosis: a systematic review. Lancet Infect Dis.2006; 6 (9): 570-81.

8. Raqib R, Mondal D, Karim MA, Chowdhury F,Ahmed S, Luby $\mathrm{S}$, et al. Detection of Antibodies Secreted from Circulating Mycobacterium tuberculosis-Specific Plasma Cells in the Diagnosis of Pediatric Tuberculosis. Clin Vaccine Immunol. 2009; 16 (4 ):521-27

9. Updated Guidelines for Using Interferon Gamma Release Assays to Detect Mycobacterium tuberculosis Infection - CDC, United States, 2010. Morbidity and Mortality Weekly Report. Recommendations and Reports. June 25, 2010 / Vol. 59 / No. RR-5.

10.Dinnes J, Deeks J, Kunst H, Gibson A, Cummins E, Waugh N, et al. A systematic review of rapid diagnostic tests for the detection of tuberculosis infection. Health Technol Assess.2007; 11 (3): 1-314.

11. Kapisyzi P, Argjiri D, Aliko A, Beli J, Vakeflliu Y, Kore R, et al. The Use of Different Cut off Values of ADA Liquid Level in Diagnosis of Tuberculous Pleurisy in Countries With Different Incidence of Tuberculosis. Chest. 2011; 140(4): 703A.

12. Helb D, Jones M, Story E, Boehme C, Wallace E, Ho K, et al. Rapid Detection of Mycobacterium tuberculosis and Rifampicin Resistance by Use of On-Demand, Near-Patient Technology. J. Clin. Microbiol. 2010; 48(1): 229-37

13. "Roadmap for rolling out Xpert MTB/RIF for rapid diagnosis of TB and MDR TB", WHO, Geneva, 8 December 2010.

14. Trebucq A, Enarson DA, Chiang CY, Deun AV, Harries AD, Boillot $\mathrm{F}$, et al. Xpert MTB/RIF for national tuberculosis programmes in low income countries: when, where and how?. Int J Tuberc Lung Dis.2011; 15(12):1567-71.

15. Verma SK, Dubey AL, Singh PA, Tewerson SL, Sharma D. Adenosine Deaminase (ADA) Level in Tubercular Pleural Effusion. Lung India. 2008; 25(3): 109-10.

16. Boehme CC. Rapid molecular detection of tuberculosis and rifampicin resistance. N Eng J of Med. 2010; 363(11):1005-15. 